\title{
The Clusters AgeS Experiment (CASE). III. Analysis of the Eccentric Eclipsing Binary V32 in the Globular Cluster NGC $6397^{1}$
}

\author{
J. Kaluzny ${ }^{2}$, I. B. Thompson ${ }^{3}$, S. M. Rucinski ${ }^{4}$, W. Krzeminski ${ }^{5}$,
}

\begin{abstract}
We present spectroscopic and photometric observations of the eclipsing binary V32 located in the central field of the globular cluster NGC 6397. The variable is a single-line spectroscopic binary with an orbital period of $9.8783 \mathrm{~d}$ and a large eccentricity of $e=0.32$. Its systemic velocity $\left(\gamma=20.7 \mathrm{~km} \mathrm{~s}^{-1}\right)$ and metallicity $([\mathrm{Fe} / \mathrm{H}] \sim-1.9)$ are both consistent with cluster membership. The primary component of the binary is located at the top of the main-sequence turn-off on the cluster color-magnitude diagram. Only a shallow primary eclipse is observed in the light curve. Based on stellar models for an age of $12 \mathrm{Gyr}$ and the mass-function derived from the radial velocity curve, we estimate the masses to be $M_{p}=0.79 \mathrm{M}_{\odot}$ and $M_{s}=0.23 \mathrm{M}_{\odot}$. The light curve of $\mathrm{V} 32$ can be reproduced by adopting $R_{p}=1.569 \mathrm{R}_{\odot}$ and $R_{s}=0.236 \mathrm{R}_{\odot}$ for the radii and $i=85.44 \mathrm{deg}$ for the system inclination. The system geometry precludes observations of the secondary eclipse. The large eccentricity of the orbit is puzzling given that for metal poor, halo binaries the transition from circular to eccentric orbit occurs at an orbital period of about 20 days. We suppose that the orbit of V32 was modified relatively recently by dynamical interaction with other cluster star(s). An alternative explanation of the observed eccentricity calls for the presence of a third body in the system.
\end{abstract}

Subject headings: binaries: spectroscopic - stars: individual (V32-NGC 6397)

\footnotetext{
${ }^{2}$ Copernicus Astronomical Center, Bartycka 18, 00-716 Warsaw, Poland; jka@camk.edu.pl

${ }^{3}$ Carnegie Observatories, 813 Santa Barbara St., Pasadena, CA 91101-1292; ian@ociw.edu

${ }^{4}$ David Dunlap Observatory, Department of Astronomy and Astrophysics, University of Toronto, P.O. Box 360, Richmond Hill, ON L4C 4Y6, Canada; rucinski@astro.utoronto.ca

${ }^{5}$ Las Campanas Observatory, Casilla 601, La Serena, Chile; wojtek@lco.cl

${ }^{1}$ This paper utilizes data obtained with the 6.5-meter Magellan Telescopes located at Las Campanas Observatory, Chile.
} 


\section{INTRODUCTION}

Little is known about the properties of main-sequence binary stars in globular clusters. These systems offer the potential for measurements of cluster age and distance independent of cluster main-sequence fitting (see, for example, Paczyński 1997; Thompson et al. 2001), and as testbeds for theories of the stellar evolution of Population-II stars.

The eclipsing binary V32-N6397 (hereafter V32) was discovered by Kaluzny et al. (2006) during a survey for variable stars in the central field of the globular cluster NGC 6397. They observed only one shallow eclipse event with a depth of about 30 mmag. With $V_{\max }=16.13$ the variable is located at the very top of the cluster main sequence, and is a candidate member of NGC 6397. In this paper we report results of follow-up photometric and spectroscopic observations of V32. Section 2 describes new photometry and a measurement of the spectroscopic orbit of V32. A self-consistent model of the system is presented in Section 3 , Finally, Section 4 briefly discusses the implications of the large measured eccentricity of the orbit of V32.

\section{SPECTROSCOPIC AND PHOTOMETRIC OBSERVATIONS}

Spectroscopic observations of V32 stars were carried out with the MIKE echelle spectrograph (Bernstein et al. 2003) on the Magellan II (Clay) telescope at Las Campanas Observatory. The data were collected during several observing runs between 2005 May and 2007 August. For this analysis we use data obtained with the blue channel of MIKE covering the wavelength range $3350 \AA$ to $5000 \AA$ at a resolving power of $\lambda / \Delta \lambda \approx 38,000$. All of the observations were obtained with a $0.7 \times 5.0$ arcsec slit and with $2 \times 2$ pixel binning. At $4380 \AA$ the resolution was $\simeq 2.7$ pixels with a scale of $0.043 \AA /$ pixel. The spectra were first processed using a pipeline developed by Dan Kelson following the formalism of Kelson (2003) and then analyzed further using standard tasks in the IRAF/Echelle package6. Each of the final individual spectra typically consisted of two $300 \mathrm{~s}$ exposures interlaced with an exposure of a thorium-argon lamp. We obtained 16 spectra of V32.

Velocities were measured with the IRAF FXCOR package. For the template we used a single MIKE spectrum of the metal-poor subgiant HD $193901([\mathrm{Fe} / \mathrm{H}]=-1.22$, Tomkin et al. 1992) with an adopted radial velocity of $-172 \mathrm{~km} \mathrm{~s}^{-1}$. The velocity measurements were made over the wavelength range $4000 \AA-5000 \AA$ with the Balmer lines masked out of the

\footnotetext{
${ }^{6}$ IRAF is distributed by the National Optical Astronomy Observatories, which are operated by the Association of Universities for Research in Astronomy, Inc., under cooperative agreement with the NSF.
} 
template and object spectra. There was no evidence for a second velocity peak in any of the object spectra and we conclude that the variable is a single-line spectroscopic binary. The observations are presented in Table 1 which lists the heliocentric Julian Date (HJD) at mid-exposure, the velocities of the primary and errors of these velocities as returned by the FXCOR routine.

The measured radial velocities of the primary were fitted with a non-linear least squares solution of the eccentric orbit using code written by G. Torres. The derived orbital elements are listed in the second column of Table 2. The quantity $T_{1}$ is the moment of periastron passage and the remaining quantities have their standard meaning. The solution with 6 free parameters implies that a superior conjunction occured at $H J D=2453109.7977 \pm 0.083$. This is consistent with with the timing of the eclipse observed on the night of 2004 April 11. Using photometry from Kaluzny et al. (2006) supplemented with new observations (see below) we estimate that the observed eclipse was centered at $H J D=2453109.829$. We then fitted the radial velocity curve by fixing the orbital period at the value which exactly predicts the moment of superior conjunction. By fitting 5 parameters we obtained $P=9.8779 \mathrm{~d}$ and $T_{1}=2453899.8248$. This ephemeris predicts correctly the timing of the eclipse observed in the 2004 season. However, it leads to unacceptable phasing of some observations from the 2007 season which were obtained shortly before ingress to the primary eclipse. The shortest orbital period which gives a symmetric primary eclipse is $P=9.8783 \mathrm{~d}$. For this period the observed moment of the observed superior conjunction can be reproduced for $T_{1}=2453899.8890$. The solution with $P$ and $T_{1}$ fixed at these values is listed in the third column of Table 2, The corresponding velocity curve and observed velocities are plotted in Fig. 1.

At the beginning of the 2007 observing season we had in hand an approximate ephemeris for V32 and further photometric observations of a few predicted eclipses of the binary were obtained. The observations were made with the TEK5 camera on the $2.5 \mathrm{~m}$ du Pont telescope at Las Campanas Observatory. The instrumental setup and reduction methods were the same as these described in Kaluzny et al. (2006). These observations were mostly hampered by poor weather but on one of the nights we obtained some useful data covering the phases immediately preceding the predicted ingress into primary eclipse. These observations helped to constrain the spectroscopic solution as described above. The $V$-band light curve based on all available observations of V32 is shown in Fig. 2 for the region of the light curve near the observed eclipse. It is phased with the spectroscopic ephemeris listed in the third column of Table 2, The continuous line shows the synthetic light curve corresponding to the model presented in the next section. The full $V$-band observation data set is given in Table 3 , The $V / B-V$ color-magnitude diagram for NGC 6397 based on the du Pont photometric observations is presented in Fig. 3, 


\section{Properties of V32}

A direct determination of the absolute parameters of the components of V32 is hampered by the lack of velocity observations of the secondary. In the following analysis we assume that the variable is a member of the globular cluster NGC 6397. This conclusion is based on four arguments. First, the systemic velocity of V32 $\left(\gamma=20.65 \pm 0.19 \mathrm{~km} \mathrm{~s}^{-1}\right)$ agrees with the mean velocity of the cluster determined by Meylan \& Mayor (1991), $V_{\text {rad }}=18.1 \pm 0.1 \mathrm{~km} \mathrm{~s}^{-1}$ with a central velocity dispersion of $4.5 \mathrm{~km} \mathrm{~s}^{-1}$. Second, on the $V / B-V$ diagram shown in Fig. 3 the binary is located at the top of the cluster turnoff, the primary is apparently just beginning to evolve onto the cluster sub-giant branch. Third, the variable is located on the sky only 51 arcsec from the projected center of NGC 6397. The cluster itself has a half-light radius of $r_{h}=140$ arcsec (Harris 1996). Finally, we have estimated the metallicity of V32. We shifted the individual spectra of V32 to zero velocity, and averaged these shifted spectra to produce a mean spectrum for V32. We measured the equivalent widths of $10 \mathrm{Fe}$ I lines selected from Thompson et al. (2008) in this mean spectrum, and compared these to the equivalent widths measured in a grid of alpha-enhanced synthetic spectra from Coelho et al. (2005) linearly interpolated at $T_{\text {eff }}=6400$ and $\log g=4.3$ for metallicities of $[\mathrm{Fe} / \mathrm{H}]=-2.4$, -2.0, -1.6, and -1.2. From this comparison we obtain $[\mathrm{Fe} / \mathrm{H}]=-1.9 \pm 0.1$ (internal standard deviation) for V32. Recent measurements of the metallicity of NGC 6397 find $[\mathrm{Fe} / \mathrm{H}]=-2.0$ (Gratton et al. 2003; Thévenin et al. 2001). While our measurement is crude (a fine analysis of the mean spectrum is in preparation (McWilliam et al. 2008)), the estimated metallicity of V32 is consistent with cluster membership.

We adopt a cluster metallicity of $[\mathrm{Fe} / \mathrm{H}]=-2.0$ with an $\alpha$-element enhancement of $[\alpha / \mathrm{H}]=+0.34$. Recent estimates of the age of NGC 6397 span a range of $11.47 \pm 0.47$ Gyr (Hansen et al. 2007) to $13.5 \pm 1.1$ (Gratton et al. (2003); a determination for models with diffusion). We adopt an age of $12.0 \pm 0.5 \mathrm{Gyr}$. Using the Dartmouth stellar model isochrones of Dotter et al. (2007) for an age of $12.0 \pm 0.5 \mathrm{Gyr},[\mathrm{Fe} / \mathrm{H}]=-2.0$ and $[\alpha / \mathrm{H}]=+0.4$, the location of the primary of $\mathrm{V} 32$ on the cluster $V /(B-V)$ diagram implies a primary mass of $M_{p}=0.790 \pm 0.009 \mathrm{M}_{\odot}$. Assuming for the moment an orbital inclination of $i=90 \mathrm{deg}$, we use the measured mass function to derive a secondary mass of $M_{s}=0.232 \pm 0.003 M_{\odot}$. The Dotter et al. (2007) models then suggest a secondary radius of $R_{s}=0.236 R_{\odot}$.

We fit the light curve of V32 using the PHOEBE code (Prša \& Zwitter 2005) which is based on the models of Wilson \& Devinney (1971) and Wilson (1979). Two free parameters were fit: the inclination $i$ and the radius of the primary. The remaining parameters were fixed as follows: The orbital elements were adopted from the 3rd column of Table 2 . The effective temperature of the primary was set at $T_{p}=6490 \mathrm{~K}$ based on an unreddened $(B-V)_{p}=0.368$ and the empirical calibration of Worthey \& Lee (2006). Following Gratton et al. (2003) we 
adopted $E(B-V)=0.183$. The temperature of the secondary was set at $T_{s}=3874$ using the Dotter et al. (2007) models. The solution converged to $i=84.96 \mathrm{deg}$ and $R_{p}=1.53 R_{\odot}$. We then made one more iteration of the above procedure starting with $i=84.96 \mathrm{deg}, M_{s}=$ $0.233 M_{\odot}$ and $R_{s}=0.237 R_{\odot}$. The solution converged to $i=85.03 \mathrm{deg}$ and $R_{p}=1.53 R_{\odot}$. The calculated luminosity ratio in the $V$-band is $L_{p} / L_{s}=664$. In the middle of the primary eclipse the entire disk of the secondary is projected against disk of the primary - the eclipse is a transit. The secondary (occultation) eclipse is not observable. The estimated radius of the primary is consistent with its location above the main-sequence turnoff on the colormagnitude diagram of the cluster. For a mass of $0.790 M_{\odot}$ and an age of 12 Gyr the models of Dotter et al. (2007) predict a radius of $r=1.40 R_{\odot}$. For the same age the radius reaches $r=1.54 R_{\odot}$ for a mass of $0.793 M_{\odot}$. Given the approximate nature of our analysis we consider the derived solution to be self-consistent.

Clearly the limited data make a direct and accurate determination of the absolute parameters for components of V32 impossible. The light curve lacks a secondary eclipse and the photometric coverage of the primary eclipse is not complete. However, the deduced parameters of V32 reproduce well its observed radial velocity and light curves, and are self-consistent with evolutionary models of low mass stars.

\section{Discussion}

The properties of V32 are consistent with membership in the globular cluster NGC 6397. The system is composed of two main-sequence stars with an age of about 12 Gyr. The orbit shows a large eccentricity with $e=0.32$. Such an eccentricity is unexpected is since tidal forces should circularize the orbit of the binary on a short time scale. The theoretical and observational aspects of the circularization of binary orbits for solar mass binaries are discussed in some detail in Meibom \& Mathieu (2005). They estimate, based on results of Latham et al. (2002), that halo binaries with periods shorter than $15.6_{-3.2}^{+2.3} \mathrm{~d}$ should have circular orbits. For the old open clusters NGC 188 (age $\approx 7$ Gyr) and M67 (age $\approx 4$ Gyr) circularization has occured for periods shorter than $14.5 \mathrm{~d}$ and $12.1 \mathrm{~d}$, respectively.

Very little is known about globular cluster binaries with orbital periods longer than a few days. Several surveys for spectroscopic binaries among cluster giants were conducted in 1980s and 1990s. They led to the detection of some candidates but generally spectroscopic orbits of these stars remain undetermined (Yan \& Cohen 1996, see also references therein). Four eclipsing binaries with periods $4<P<10$ days were detected in the central part of 47 Tuc in the HST/WFPC2 survey by Albrow et al. (2001) using photometric observations. All of these have presumed circular orbits although observations do not cover the full orbital 
cycle for two of the systems with the longest periods. Extensive ground base surveys of 47 Tuc (Weldrake et al. 2004) and $\omega$ Cen (Kaluzny et al. 2004; Weldrake et al. 2007) led to the detection of two eclipsing binaries with orbital periods exceeding 10 days. Follow up observations conducted by our group show that 47 Tuc-V69 and $\omega$ Cen-V406 have eccentric orbits with $P=29 \mathrm{~d}$ and $P=71 \mathrm{~d}$, respectively (Thompson et al. 2008, in preparation). All of the other cluster eclipsing binaries reported so far have orbital periods less than 10 days and show circular orbits.

The eccentric orbit of V32 can possibly be due to a relatively recent dynamical interaction of the binary with other cluster star(s). NGC 6397 has a "collapsed" core (Diorgovski \& King 1986) containing a population of about 20 X-ray sources including 9 candidate cataclysmic variables, a millisecond pulsar and several candidate BY Dra-type close binaries (Grindlay et al. 2001). Kaluzny et al. (2006) reported the detection of 9 eclipsing binaries and 6 candidates for ellipsoidal binaries located in the central part of the cluster. A dynamical interaction capable of transforming the circular orbit of V32 into a highly eccentric one would also likely significantly disturb the systemic velocity of the binary. Our data show that the radial velocity of V32 is not unusual for an object from the central part of the cluster. Unfortunately, the transverse velocity is V32 is unknown as the binary is not included in the recent proper motion survey of NGC 6397 conducted by Kalirai et al. (2007).

As an alternative explanation of the eccentric orbit of V32 we consider the possibility that the system is in fact a hierarchical triple. The third component located on the outer orbit is capable of generating eccentricity in the inner binary. Extensive reviews of the evolution of the orbits of binary stars have been presented by Eggleton (2006) and Mazeh (2008). Numerical simulations of stellar clusters predict dynamical formation of triple stars, especially in the presence of primordial binaries (McMillan et al. 1990; Heggie \& Aarseth 1992). Examples of known triple stars in globular clusters include the pulsar PSR B162026 in M4 (Thorsett et al. 1993) and possibly the ultracompact X-ray binary 4U 1820-303 (Zdziarski et al. 2007). The hypothesis that V32 is a triple can be tested by spectroscopic observations aimed at detection of variability of the systemic velocity of the putative inner binary. We see no systemic velocity residuals from the orbit give in Table 3 over the 2.2 year duration of our velocity observations.

Finally we comment on possibility that the eccentric orbit of V32 is related to its rather low mass ratio $q=M_{s} / M_{p} \approx 0.30$. According to Mathieu \& Mazeh (1988) the time scale of circularization of a binary is related to $q$ by the relation: $\tau_{\text {circ }} \propto q^{2 / 3}\left(1+q^{-1}\right)^{5 / 3}$. This corresponds to an increase of $\tau_{\text {circ }}$ of a factor of 1.6 as the mass ratio ranges from $q=1$ to $q=0.3$. For low values of $q$ the contribution of the secondary to the circularization process becomes negligible (Mazeh 2008). At the same time $\tau_{\text {circ }}$ is a strong function of the orbital 
period $P$, with $\tau_{\text {circ }} \propto P^{16 / 3}$ (Zahn 1977). The net result is that the circularization period will lengthen as the mass ratio decreases from for $q=1$ and $q=0.3$, especially for long period systems. It is appropriate to note at this point that that the sample of halo stars presented by Latham et al. (2002) consists entirely of single line binaries and hence objects with mass ratios noticeably lower than unity. More globular cluster binaries with period of the order of 10 days have to be detected and analyzed before V32 can be called a truly unusual system. The CASE group is in a process of collecting data which can shed more light on the relation between eccentricity and orbital period for binaries in globular clusters.

JK and WK were supported by the grant 1 P03D 00128 from the Ministry of Science and Higher Education, Poland. Research of JK is also supported by the Foundation for Polish Science through the grant MISTRZ. IBT was supported by NSF grant AST-0507325. Support from the Natural Sciences and Engineering Council of Canada to SMR is acknowledged with gratitude. It is a pleasure to thank Willy Torres for sharing his code with us.

\section{REFERENCES}

Albrow, M.D., Gilliland, R.L., Brown, T.M., Edmonds, P.D., Guhathakurta, P., \& Sarajedini, A 2001, ApJ, 559, 1060

Bernstein, R., Shectman, S. A., Gunnels, S. M., Mochnacki, S., \& Athey, A. E. 2003, Instrument Design and Performance for Optical/Infrared Ground-based Telescopes. Edited by Iye, Masanori; Moorwood, Alan F. M. Proceedings of the SPIE, 4841, 1694

Coelho, P., Barbuy, B., Meléndez, J, Schiavon, R.P., \& Castilho, B.V. 2005, A\&A, 443, 735

Djorgovski, S., \& King, I.R. 1986, ApJ, 305, L61

Dotter, A., Chaboyer, B., Jevremovi, D., Baron, E., Ferguson, J. W., Sarajedini, A., Anderson, J. 2007, AJ, 134, 376

Eggleton, P. 2006, Evolutionary Processes in Binary and Multiple Stars, Cambridge Astrophysics Series, No. 40

Gratton, R.G., Bragaglia, A., Carretta, E., Clementini, G., Desidera, S., Grundahl, F., \& Lucatello, S. 2003, A\&A, 408, 529

Grindlay, J.E., Heinke, C.O., Edmonds, P.D., Murray, S.S., \& Cool, A.M. 2001, ApJ, 563, 53 
Harris, W. E. 1996, AJ, 112, 1487

Heggie, D.C., \& Aarseth, S.J. 1992, MNRAS, 257, 513

Hansen, B. M. S., et al. 2007, ApJ, 671, 380

Kaluzny, J., Olech, A., Thompson, I.B., Pych, W., Krzeminski, W., Schwarzenberg-Czerny, A. 2004, A\&A, 424, 1101

Kaluzny, J., Thompson, I.B., Krzeminski, W., Schwarzenberg-Czerny, A. 2006, MNRAS, 365,548

Kalirai, J.S., et al. 2007, ApJ, 657, L93

Kelson D. D. 2003, PASP, 115, 688

Latham, D.W. et al. 2002,AJ, 124, 1144

Mathieu, R.D., \& Mazeh, T. 1988, ApJ, 326, 256

Mazeh, T. 2008, in Tidal Effect in Stars, Planets, and Disks, Ed. M.-J. Goupil and J.-P. Zahn, EAS Publications Series, in press, preprint arXiv:0801.0134

McMillan, S, Hutt, P, \& Makino, J. 1990, ApJ, 362, 522

Meibom, S., Mathieu, R.D. 2005, ApJ, 620, 970

Meylan, G., \& Mayor, M. 1991, A\&A,250, 113

Paczyński, B. 1997, in The Extragalactic Distance Scale STScI Symposium (Cambridge: Cambridge University Press)

Prŝa, A., Zwitter, T. 2005, ApJ, 628, 426

Thévenin, F., Charbonnel, C., de Freitas Pacheco, J. A., Idiart, T. P., Jasniewicz, G., de Laverny, P., \& Plez, B. 2001, A\&A, 373, 905

Thompson, I. B., Kaluzny, J., Pych, W., Burley, G., Krzeminski, W., Paczyński, B., Persson, S. E., \& Preston, G. W., AJ, 121, 3089

Thompson, I. B., Ivans, I. I., Bisterzo, S., Sneden, C., Gallino, R., Vauclair, S., Burley, G. S., Shectma, S. A., \& Preston, G. W. 2008, ApJ, in press, preprint arXiv:0712:3228

Tomkin, J., Lemke, M., Lambert, D.L., \& Sneden, C. 1992, AJ, 104, 1568 
Thorsett, S.E., Arzoumanian, Z., \& Taylor, J.H. 1993, ApJ, 412, L33

Weldrake, D.T.F., Sackett, P.D., Bridges, T.J., \& Freeman, K.C. 2004, AJ, 128, 736

Weldrake, D.T.F., Sackett, P.D., \& Bridges, T.J. 2007, AJ, 133, 1447

Wilson, R. E., \& Devinney, E. J. 1971, ApJ, 166, 605

Wilson, R. E. 1979, ApJ, 234, 1054

Worthey, G., \& Lee, H.-C. 2006, preprint (astro-ph/0604590)

Yan, L., \& Cohen, J.G. 1996, AJ, 112, 1489

Zahn, J.-P., 1977, A\&A, 57, 383

Zdziarski, A.A., Wen, L., \& Gierlioński, M. 2007, MNRAS, 377, 1006

Zucker, S. \& Mazeh, T. 1994, ApJ, 420, 806 
Table 1. Velocity Observations of V32

\begin{tabular}{crc}
\hline \hline $\begin{array}{c}\text { HJD } \\
(-2450000)\end{array}$ & $\begin{array}{c}\mathrm{V}_{\text {rad }} \\
\mathrm{km} \mathrm{s}^{-1}\end{array}$ & $\begin{array}{c}\sigma \\
\mathrm{km} \mathrm{s}^{-1}\end{array}$ \\
\hline 3521.85254 & 36.99 & 0.26 \\
3581.71777 & 42.49 & 0.34 \\
3582.69824 & 46.92 & 0.27 \\
3584.66211 & 06.51 & 0.28 \\
3875.88965 & 22.24 & 0.41 \\
3876.71387 & 29.18 & 0.29 \\
3877.72266 & 38.38 & 0.34 \\
3891.82129 & -1.19 & 0.33 \\
3898.77344 & 45.71 & 0.38 \\
3935.65430 & 26.52 & 0.34 \\
3938.69238 & 45.06 & 0.54 \\
3989.54590 & 07.17 & 0.37 \\
3991.58301 & 02.48 & 0.54 \\
4258.67197 & 03.67 & 0.53 \\
4259.73913 & 09.98 & 0.58 \\
4329.66700 & 16.86 & 0.43 \\
\hline
\end{tabular}


Table 2. Orbital Parameters for V32

\begin{tabular}{lrr}
\hline \hline \multicolumn{1}{c}{ Parameter } & \multicolumn{1}{c}{ Value } & \multicolumn{1}{c}{ Value } \\
\hline$P$ (days) & $9.8783 \pm 0.0011$ & $9.8783^{\text {a }}$ \\
$T_{1}\left(\mathrm{HJD}^{2} 25\right.$ 0000 $)$ & $3899.832 \pm 0.083$ & $3899.889^{\mathrm{a}}$ \\
$\gamma\left(\mathrm{km} \mathrm{s}^{-1}\right)$ & $20.67 \pm 0.22$ & $20.71 \pm 0.18$ \\
$K_{p}\left(\mathrm{~km} \mathrm{~s}^{-1}\right)$ & $23.92 \pm 0.30$ & $24.00 \pm 0.25$ \\
$e$ & $0.320 \pm 0.011$ & $0.322 \pm 0.009$ \\
$\omega($ deg $)$ & $72.7 \pm 3.0$ & $74.74 \pm 0.7$ \\
$(O-C) r m s\left(k m s^{-1}\right)$ & 0.60 & 0.62 \\
Derived quantities: & & \\
$f(M) \sin ^{3} i\left(M_{\odot}\right)$ & $0.01192 \pm 0.00046$ & $0.01201 \pm 0.00042$ \\
$A_{p} \sin (i)\left(R_{\odot}\right)$ & $3.079 \pm 0.039$ & $3.086 \pm 0.036$ \\
\hline
\end{tabular}

${ }^{a}$ Fixed based on photometry. 
Table 3. $\quad V$-band Photometric Observations of V32

\begin{tabular}{ccc}
\hline \hline $\begin{array}{c}\text { HJD } \\
(-2450000)\end{array}$ & $V$ & $\sigma_{V}$ \\
\hline 2765.8121 & 16.128 & 0.008 \\
2765.8209 & 16.134 & 0.008 \\
2765.8277 & 16.128 & 0.008 \\
2765.8371 & 16.126 & 0.008 \\
2765.8677 & 16.125 & 0.008 \\
2765.8894 & 16.129 & 0.008 \\
2765.8966 & 16.122 & 0.008 \\
2765.9061 & 16.129 & 0.008 \\
2765.9128 & 16.127 & 0.008 \\
2765.9294 & 16.131 & 0.008 \\
\hline
\end{tabular}

Note. - This Table is published in its entirety in the electronic addition of the Astronomical Journal. A portion here is shown for guidance regarding its form and content. 

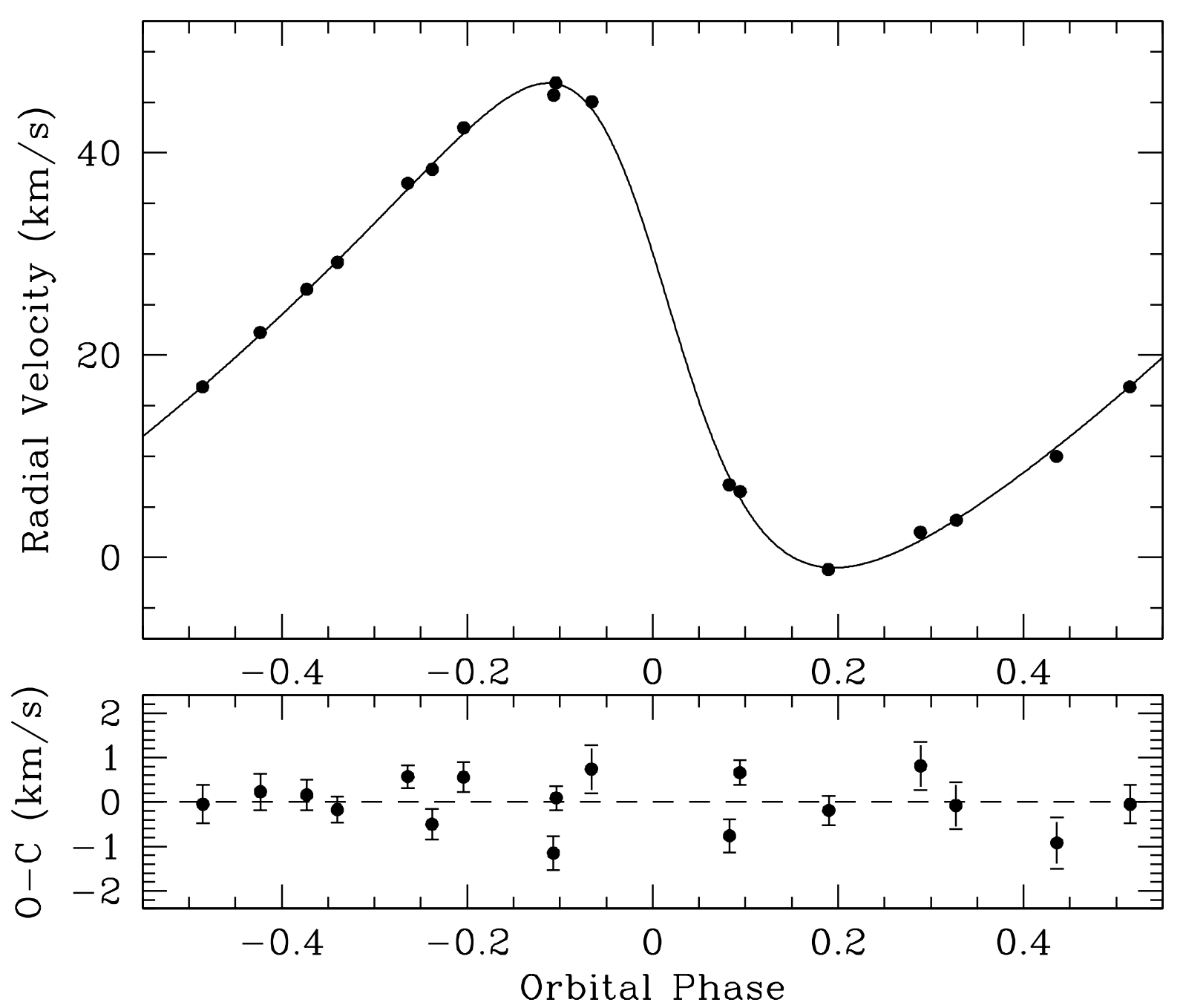

Fig. 1. - The velocity curve for orbital solution for V32, together with the individual observed velocities. The O-C residuals from the solution are shown in the lower panel. 

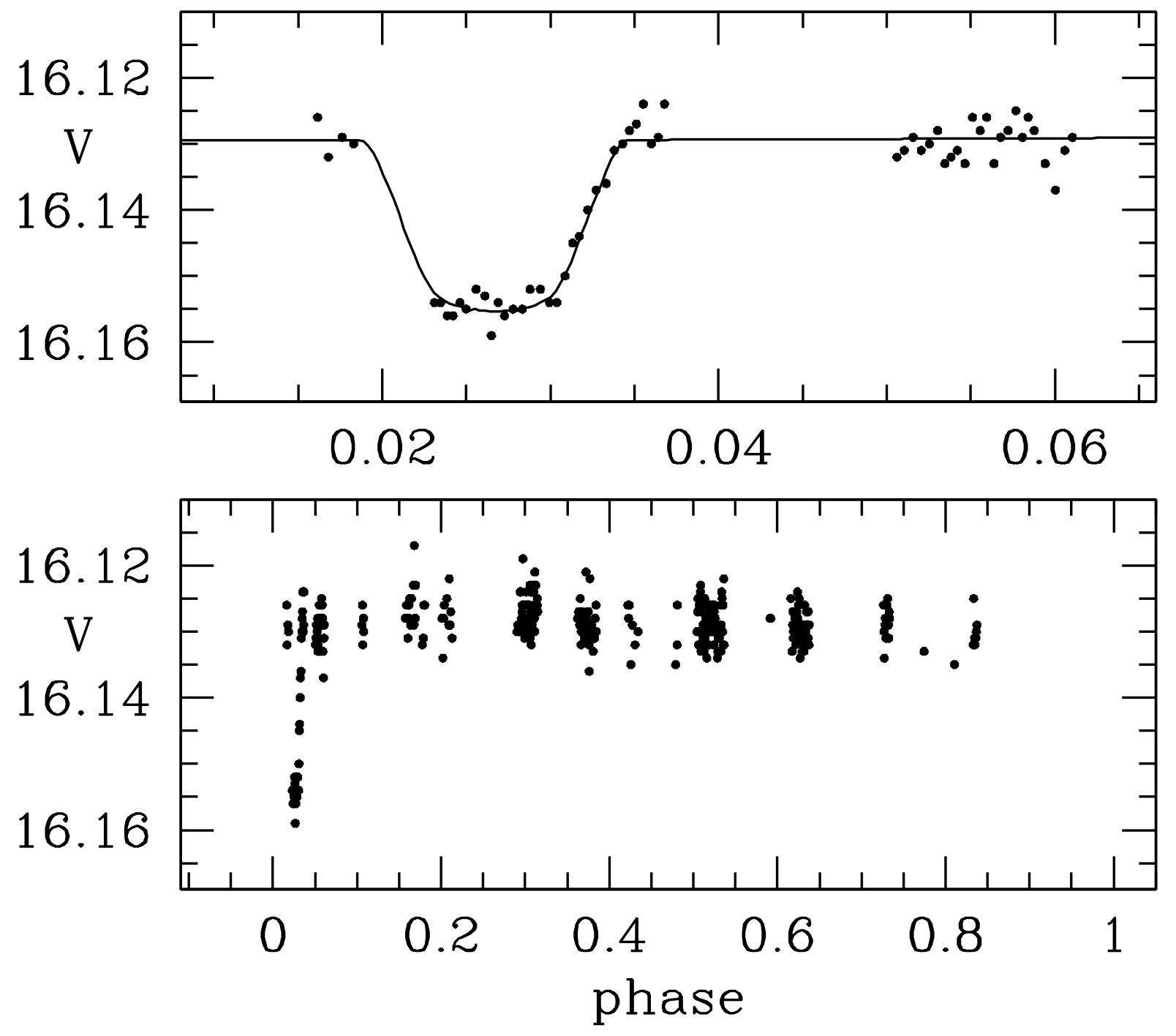

Fig. 2.- The phased observed $V$ light curve of V32. The synthetic curve corresponding to the model described in Sec. 3 is shown in the upper panel. 


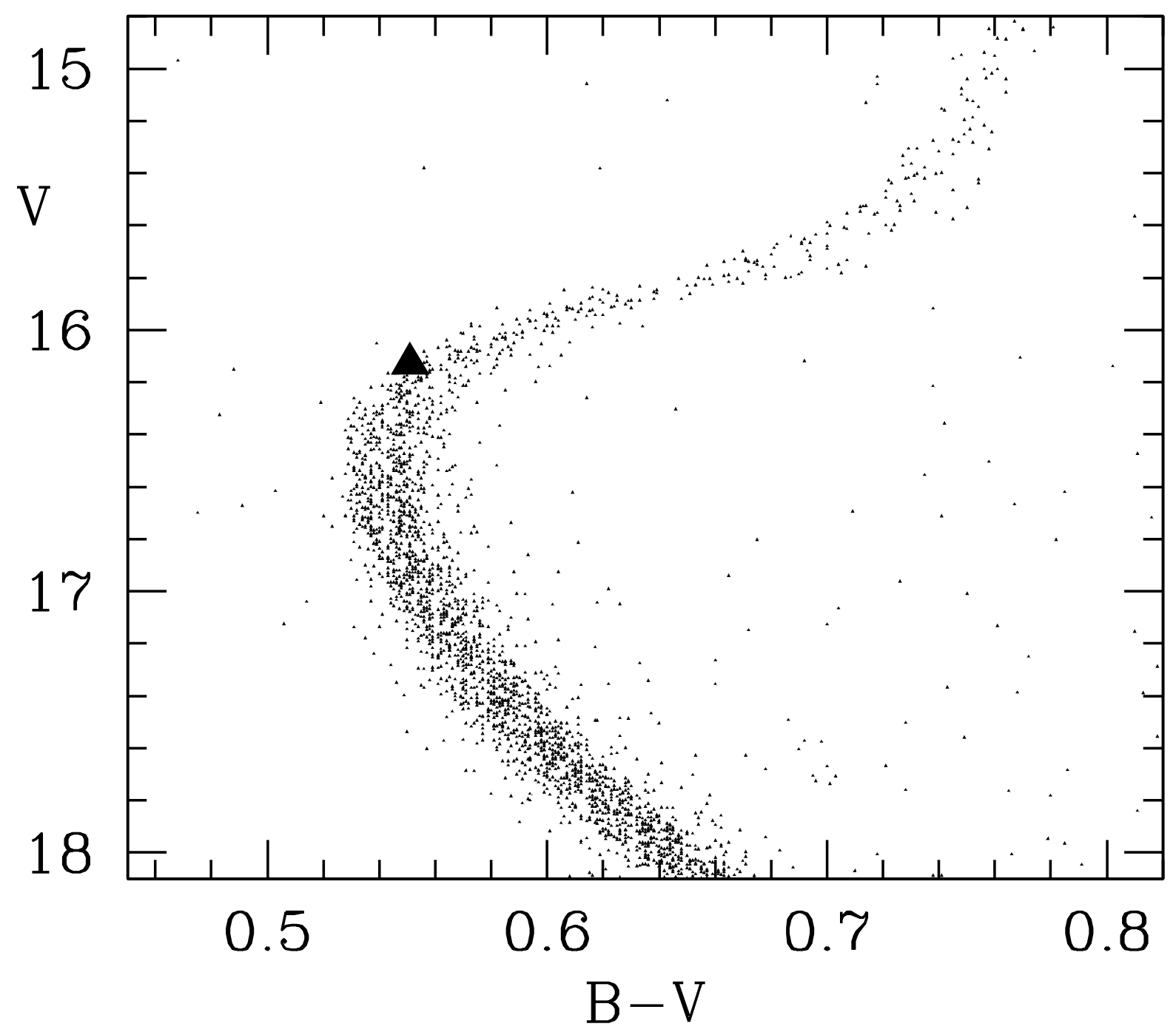

Fig. 3.- Position of V32 in the $V / B-V$ diagram for NGC 6397. 